\section{Avaliação da exposição a BTEX em postos de revenda de combustíveis no Rio de Janeiro, Brasil, e os riscos à saúde do trabalhador}

\author{
Assessment of exposure to BTEX in vehicle filling \\ stations in Rio de Janeiro, Brazil, and risks to \\ workers' health
}

\section{Evaluación de la exposición a BTEX en puestos de reventa de combustibles, en Río de Janeiro, Brasil, y riesgos para la salud del trabajador}

\author{
Victor Oliva Figueiredo 1 \\ Leandro Vargas Barreto de Carvalho 1 \\ Renato Marçullo Borges 1 \\ Isabele Campos Costa-Amaral 1 \\ Marcus Vinicius Corrêa dos Santos 1 \\ Ana Cristina Simões Rosa 1 \\ Marco Antônio Carneiro de Menezes 1 \\ Rita de Cássia Oliveira da Costa Mattos 1 \\ Paula Novaes Sarcinelli 1 \\ Sergio Rabello Alves 1 \\ Ariane Leites Larentis 1 \\ Eline Simões Gonçalves 1
}

\section{Resumo}

A gasolina é uma mistura complexa de substâncias, dentre elas estão hidrocarbonetos como o benzeno, tolueno, etilbenzeno e xilenos (BTEX), sendo esses compostos emitidos para a atmosfera, com destaque ao benzeno, por ser comprovadamente carcinogênico. Objetivamos avaliar as concentrações de BTEX no ar de postos de revenda de combustíveis no Município do Rio de Janeiro, Brasil, e calcular o risco de câncer associado a estas exposições. Foram realizados dois tipos de amostragem (fixa e móvel) adaptadas da metodologia no 1.501 do Instituto Nacional de Segurança e Saúde Ocupacional (Estados Unidos), em seis postos de revenda de combustiveis na Zona Oeste da cidade. A amostragem fixa foi feita próxima às bombas de combustível, enquanto a móvel foi realizada através da coleta do ar na zona respiratória dos trabalhadores (frentistas), conforme estes se deslocavam pelo posto. As amostras foram analisadas por cromatografia gasosa com deteç̧ão por ionização em chama. Com os resultados das amostragens foram feitos os cálculos de risco à saúde, usando os indicadores Quociente de perigo (HQ) e Risco de câncer (CR), para avaliar os possíveis efeitos não-carcinogênicos e carcinogênicos, respectivamente, nos trabalhadores dos postos de revenda de combustiveis. As concentrações ambientais para a maioria dos compostos BTEX foram abaixo dos limites preconizados, menos para o benzeno, um composto carcinogênico, que apresentou concentrações muito acima dos limites, levando a altos valores de risco de câncer. Os resultados demonstraram que há riscos à saúde dos trabalhadores de postos de revenda de combustiveis, principalmente o risco de desenvolver câncer, devido à exposição excessiva ao benzeno.

Saúde do Trabalhador; Benzeno; Posto de Combustível; Cancerígeno doi: 10.1590/0102-311X00351520

\author{
Correspondência \\ V. O. Figueiredo \\ Rua das Laranjeiras 115, Rio de Janeiro, RJ 22240-000, Brasil. \\ victorof30@hotmail.com \\ 1 Escola Nacional de Saúde Pública Sergio Arouca, Fundação \\ Oswaldo Cruz, Rio de Janeiro, Brasil.
}




\section{Introdução}

As contaminações ambientais e as exposições ocupacionais, relacionadas à gasolina e outros combustíveis, preocupam as autoridades de vigilância em saúde e atingem um grande número de trabalhadores e residentes do entorno dos postos de revenda de combustíveis 1,2 .

A gasolina é composta por uma mistura complexa de substâncias, dentre elas os hidrocarbonetos alifáticos e aromáticos, como benzeno, tolueno, etilbenzeno e xilenos - orto $(\mathrm{o})$, meta $(\mathrm{m})$ e para ( $p$ ) (BTEX), que são compostos lipossolúveis, tóxicos e agem como depressores do sistema nervoso central 3,4 .

Os postos de revenda de combustíveis possuem um grande potencial contaminante, causado pelos tanques de armazenamento, cujo vazamento pode gerar um passivo ambiental, assim como pela manipulação diária e ininterrupta dos combustíveis aos quais estão expostos trabalhadores e a população que reside no entorno 5,6 .

Nos postos de revenda de combustíveis são realizadas uma grande variedade de atividades que vão além do abastecimento de veículos, como a troca de óleo, lavagem de carros, calibração de pneus, e também a venda de alimentos (lojas de conveniência), dentre outras atividades. Essas atividades, juntamente com a elevada taxa de evaporação dos combustíveis comercializados e da emissão de gases dos motores dos veículos, são as principais fontes causadoras da baixa qualidade do ar nos postos de revenda de combustíveis e área circunvizinha 7,8 .

No Estado do Rio de Janeiro, Brasil, 328 áreas contaminadas foram mapeadas no ano de 2015, sendo que em 59\% (192) destas áreas a atividade era de postos de revenda de combustíveis. Hidrocarbonetos totais de petróleo, compostos orgânicos voláteis e semivoláteis e BTEX respondem pelos maiores contaminantes neste ramo de atividade 9 . Os BTEX são compostos altamente voláteis e similares entre si no que tange às suas características físico-químicas, como solubilidade e volatilidade, $\mathrm{e}$ também quanto às suas vias de dispersão no ambiente 10.

Quanto aos danos toxicológicos, o benzeno pode ter efeito deletério sobre os sistemas hematopoiético, central e reprodutor. Já a exposição ao tolueno está associada a danos sobre os sistemas hematopoiético e reprodutor. Níveis de exposição de etilbenzeno e xilenos estão associados a intercorrências sobre os sistemas respiratório e nervoso ${ }^{4}$. Considerando esses efeitos causados pela exposição ao BTEX, o benzeno tem maior relevância toxicológica e está inserido na lista da Agência Internacional de Pesquisa do Câncer (IARC) como uma substância reconhecidamente carcinogênica (grupo 1A) 6,11,12.

Uma das maiores ameaças à saúde é o risco de desenvolvimento de câncer, sendo um dos efeitos toxicológicos mais perigosos do BTEX, principalmente o benzeno. Foi observado que pessoas ocupacionalmente expostas apresentam um aumento na incidência de leucemia, principalmente a leucemia mieloide aguda 13. Através do uso de modelos matemáticos, baseados em estudos em humanos e animais, é possível estimar a probabilidade de uma pessoa vir a desenvolver um câncer por respirar ar contendo concentrações específicas de compostos químicos 14 .

A Organização Mundial da Saúde (OMS) 14 estabelece que a exposição a $1 \mu \mathrm{g} \mathrm{m}-3$ de benzeno por um período de 15 anos pode levar ao aparecimento de seis casos de leucemia em uma população de 1 milhão de habitantes, pois a intoxicação por BTEX, especialmente o benzeno, é gradativa e cumulativa, já que esses compostos são metabolizados e excretados lentamente pelo organismo 15.

A exposição ocupacional de trabalhadores em relação ao BTEX pode ocorrer na manipulação direta do combustível, nos processos de síntese de derivados do petróleo e nas atividades que liberaram gases e vapores. Devido a isso, diversas estratégias são implantadas no sentido de diminuir a exposição e respectivo risco, de maneira a garantir o menor impacto à saúde desses trabalhadores 8 . Uma dessas estratégias é medir a exposição ao BTEX através da avaliação ambiental, e de risco à saúde do indivíduo, monitorando a concentração de solventes na zona respiratória e comparando com os limites de exposição ocupacional preestabelecidos 3 .

O objetivo deste trabalho foi avaliar a exposição ocupacional ao BTEX em postos de revenda de combustíveis no Município do Rio de Janeiro, através da técnica de amostragem ativa e executar, concomitantemente, a avaliação de risco, com o cálculo de risco de câncer e quociente de perigo, realizado para cada posto. 


\section{Métodos}

\section{Área de estudo}

Os seis postos de revenda de combustíveis avaliados neste estudo estão localizados nos bairros de Santa Cruz, Paciência e Sepetiba, inseridos na área de planejamento da Zona Oeste (AP 5.3), no extremo oeste do Município do Rio de Janeiro.

A AP 5.3 é considerada a segunda mais populosa do município, com mais de 1,5 milhão de habitantes, que corresponde a $26,6 \%$ da população do município. A região caracteriza-se por ser de clima quente e úmido, com temperaturas médias variando de $20^{\circ} \mathrm{C}$ a $27^{\circ} \mathrm{C}$, sendo os meses mais quentes compreendidos entre novembro e abril, e os mais frios entre maio e outubro. As chuvas são mais frequentes entre os meses de dezembro e março, sendo janeiro o mais chuvoso, e o período mais seco de junho a setembro 16 .

O estudo contou com a participação de trabalhadores (frentistas) que atuavam no turno da manhã nos postos de revenda de combustíveis. Todos os participantes receberam orientações sobre a pesquisa e assinaram voluntariamente o Termo de Consentimento Livre e Esclarecido (TCLE). Os critérios de inclusão adotados foram: ter idade superior a 18 anos, podendo ser de ambos os sexos, e ter aceitado participar do estudo através da assinatura do TCLE, sendo excluídas as mulheres grávidas, pois, na época em que o estudo foi realizado, não havia ocorrido a revisão das normas regulamentadoras, logo, as mulheres grávidas não podiam ficar em um local insalubre, como é o ambiente de um postos de revenda de combustíveis. Este estudo foi aprovado pelo Comitê de Ética em Pesquisa da Fundação Oswaldo Cruz (CAAE 17438013.5.0000.5240).

\section{Amostragem}

Os métodos de coleta e determinação de BTEX no ar adotados para a realização deste estudo foram baseados na metodologia no 1.501 do manual de métodos analíticos do Instituto Nacional de Segurança e Saúde Ocupacional dos Estados Unidos (NIOSH) 17.

A coleta foi realizada com amostradores ativos nos locais de trabalho (postos de revenda de combustíveis), entre os meses de fevereiro a junho de 2015.

Foram adotadas duas estratégias de amostragem ativa de ar ambiente nos postos: uma amostragem fixa e outra, móvel. Em ambas as coletas foram utilizadas bombas de baixo volume (modelo PCXR4; SKC Inc., https://www.skcinc.com/) e tubos preenchidos com carvão ativado em duas seções de 100 e 50mg (Anasorb CSC 226-01; SKC Inc.).

$\mathrm{Na}$ amostragem fixa, as bombas foram posicionadas próximas à área de circulação dos trabalhadores e às bombas de combustíveis, em uma altura próxima da zona respiratória, a cerca de $1,50 \mathrm{~m}$ do solo.

$\mathrm{Na}$ amostragem móvel, as bombas de amostragens ficaram presas na cintura dos trabalhadores (amostragem pessoal), e os tubos de carvão ativo foram posicionados na lapela dos uniformes, ficando próximos à zona respiratória dos frentistas. As coletas duraram em média 5 horas, com fluxo de bombeamento de $0,2 \mathrm{~L} \mathrm{~min}-1$ e volume total de $30 \mathrm{~L}$ ou $0,03 \mathrm{~m}^{3}$. Durante o período de coleta, foram feitas de 4 a 5 medidas da temperatura do ambiente, com um termômetro digital devidamente calibrado, para o cálculo da temperatura média de cada posto.

\section{Determinação de BTEX no ar}

A extração de BTEX a partir do adsorvente dos amostradores utilizados na amostragem ativa foi feita com a adição de $1 \mathrm{~mL}$ de dissulfeto de carbono com baixo teor de benzeno (Sigma-Aldrich, https:// www.sigmaaldrich.com), em vial contendo o adsorvente (contido nas duas seções do tubo de amostragem ativa), e deixado em agitação (Vortex Biomixer QL-901; http://www.biosystems.com.br/) por 60 minutos. Após este período, retirou-se o extrato com pipeta Pasteur, previamente descontaminada em mufla por 4 horas a $400^{\circ} \mathrm{C}$, procurando-se evitar que parte do adsorvente fosse aspirada neste processo. 
Para a identificação e quantificação de BTEX nas amostras foi utilizada a técnica analítica de cromatografia em fase gasosa com detecção por ionização em chama (GC-FID, Focus; Thermo-Finnigan, https://www.thermofisher.com). A metodologia de análise usou coluna capilar Carbowax 20M (Agilent, https://www.agilent.com/) com $60 \mathrm{~m}$ de comprimento, 0,32mm de diâmetro e 0,25 $\mu \mathrm{m}$ de espessura de filme.

Os parâmetros utilizados na detecção por GC-FID foram: modo de injeção Split, fluxo de injeção de $10 \mathrm{~mL} \mathrm{~min}-1$, com temperatura do injetor de $240^{\circ} \mathrm{C}$, nitrogênio como gás carreador e fluxo de $1,0 \mathrm{~mL} \mathrm{~min}-1$, temperatura do detector de $240^{\circ} \mathrm{C}$ e o volume de injeção foi de $2 \mu \mathrm{L}$.

A quantificação de BTEX foi realizada por padronização externa através da construção de uma curva analítica na matriz da amostra, ou seja, no extrato obtido a partir da extração de brancos de cartucho. Os padrões analíticos de benzeno, tolueno, etilbenzeno e xilenos $(o, m$ e $p$ ) foram da marca Sigma-Aldrich, com pureza acima de $99,9 \%$. Os pontos da curva analítica compreenderam a faixa de 0,5 a $10 \mathrm{mg} \mathrm{mL}^{-1}$. Para a discussão dos resultados de $o, m$ e $p$-xileno, os valores foram somados e apresentados como concentração de xileno total.

\section{Análise estatística}

A análise descritiva foi realizada utilizando médias, desvios padrão, frequência e percentual de distribuição. A normalidade de distribuição das variáveis contínuas foi testada por meio do teste Kolmogorov-Smirnov, adotando nível de significância de 5\%. Para comparação de variáveis com distribuição normal foram utilizados os testes t de Student ou Anova e para variáveis com distribuição não normal, os testes Mann-Whitney (U) ou Kruskal-Wallis (H).

\section{Avaliação de riscos à saúde}

Algumas metodologias de avaliação de risco são utilizadas para medir os possíveis efeitos carcinogênicos e não-carcinogênicos de compostos químicos sobre a saúde humana 17,18. Desta forma, buscando-se avaliar o risco dos trabalhadores à exposição ocupacional ao BTEX, foram calculados a partir das concentrações encontradas neste estudo, considerando 25 anos de exposição, baseado no estudo de Edokopolo et al. ${ }^{19}$, os seguintes indicadores de risco à saúde: Risco de câncer - CR (apenas para benzeno) e Quociente de perigo - HQ (para BTEX).

Para o cálculo do CR e HQ foi necessário calcular a dose média diária da exposição (LADD) ao BTEX 2,20, conforme a Equação 1.

$$
\mathrm{LADD}=(\mathrm{C} \times \mathrm{IR} \times \mathrm{EL} \times \mathrm{ED}) /(\mathrm{BW} \times \mathrm{LT})
$$

Em que LADD representa a dose média diária da exposição ( $\mu \mathrm{g}$ kg-1/dia); C é a concentração do composto; IR é a razão da inalação; EL é o alcance da exposição; ED é a duração da exposição (conversão de anos para dias: 7 dias/semana x 52 semanas/ano x 25 anos = 9.100 dias); BW é o peso corporal; e LT é o tempo de vida (idade, conversão de anos para dias: 7 dias/semana $\times 52$ semanas/ano $\times 70$ anos $=$ 25.480 dias). Estes parâmetros são sistematizados na Tabela 119.

O CR indica o excesso de risco de vir a desenvolver câncer durante um período de exposição a uma substância química 20, e foi calculado usando a Equação 2.

$$
\mathrm{CR}=\mathrm{LADD} \times \mathrm{SF}
$$

Em que CR representa o risco de câncer, e SF, o slope factor ou inclinação, expresso em ( $\mu \mathrm{g} \mathrm{kg-1/}$ dia)-1, que é um número único calculado observando os dados toxicológicos de como o risco de câncer aumenta com o aumento da exposição a um determinado composto químico.

Os valores de CR podem ser classificados como de "preocupação por efeitos carcinogênicos" (valores de CR > 10-6), ou "de níveis aceitáveis" (valores de CR $\leq 10-6$ ). De acordo com a Agência de Proteção Ambiental dos Estados Unidos (USEPA) o valor limite para risco de câncer (CRVL) é de 1,0x10-6. 
Tabela 1

Parâmetros de exposição usados para o cálculo da dose média diária da exposição (LADD).

\begin{tabular}{lcc}
\hline Parâmetro & Unidade & Valor usado \\
\hline C (concentração do composto) & $\mu g \mathrm{~m}^{-3}$ & variável \\
IR (razão da inalação) & $\mathrm{m}^{3} \mathrm{~h}-1$ & 1,4 \\
EL (alcance da exposição) & $\mathrm{h} \mathrm{dia-1}$ & 8 \\
ED (duração da exposição) & dias & 9.100 \\
BW (peso corporal) & $\mathrm{kg}$ & 70 \\
LT (tempo de vida) & dias & 25.480 \\
\hline
\end{tabular}

O CR foi calculado para o benzeno utilizando SF de 0,0273( $\mu \mathrm{g} \mathrm{kg-1/dia)-1.} \mathrm{Os} \mathrm{compostos} \mathrm{tolueno,}$ etilbenzeno e xilenos não puderam ter seu risco de câncer calculado, pois não apresentam valor de SF na literatura 19 .

O HQ é um índice que visa caracterizar o risco, sendo utilizado para calcular o risco de efeitos adversos não carcinogênicos em trabalhadores expostos ao BTEX 19. Neste estudo, o HQ foi calculado conforme a Equação 3:

$$
\mathrm{HQ}=\mathrm{LADD} / \mathrm{RfD}
$$

$\mathrm{Na}$ qual LADD representa a dose média diária da exposição ( $\mu \mathrm{g} \mathrm{kg-1/dia)} \mathrm{e} \mathrm{RfD} \mathrm{é} \mathrm{a} \mathrm{dose} \mathrm{de} \mathrm{refe-}$ rência ( $\mu \mathrm{g} \mathrm{kg}^{-1} / \mathrm{dia}$ ), diferente para cada substância química, abaixo da qual não há risco de ocorrência de efeitos adversos não carcinogênicos. A RfD é utilizada para estimar o HQ da exposição ao benzeno em diferentes cenários, sendo a RfD do benzeno 0,00855mg kg-1/dia 19 .

Desta forma, medidas cujo HQ calculado está abaixo de $1(\mathrm{HQ}<1)$, indicam um efeito não-adverso aparente nos trabalhadores durante a exposição. Já os valores acima de $1(\mathrm{HQ}>1)$ indicam aparentes efeitos adversos à saúde, provenientes da exposição ao BTEX durante o processo de trabalho.

\section{Resultados}

O método analítico foi adequado à aplicação pretendida, apresentando limites de detecção (LD) e de quantificação (LQ) para o benzeno de $1,1 \mu \mathrm{g} \mathrm{m} \mathrm{m}^{-3}$ e 3,5 $\mathrm{g} \mathrm{g} \mathrm{m}^{-3}$, respectivamente; para o tolueno, LD e LQ foram $1,9 \mu \mathrm{g} \mathrm{m}-3$ e $6,5 \mu \mathrm{g} \mathrm{m}^{-3}$, respectivamente. Os limites do etilbenzeno foram $0,2 \mu \mathrm{g} \mathrm{m}^{-3}$ e $0,8 \mu \mathrm{g} \mathrm{m}^{-3}$, respectivamente; para $p$-xileno foram $2,0 \mu \mathrm{g} \mathrm{m}^{-3}$ e $6,6 \mu \mathrm{g} \mathrm{m}^{-3}$, respectivamente, para $m$-xileno foram $0,2 \mu \mathrm{g} \mathrm{m}^{-3}$ e $0,6 \mu \mathrm{g} \mathrm{m}^{-3}$, respectivamente e $o$-xileno foram $0,4 \mu \mathrm{g} \mathrm{m}^{-3}$ e $1,4 \mu \mathrm{g} \mathrm{m}^{-3}$, respectivamente.

Os resultados das concentrações de BTEX determinadas na amostragem de ar (móvel e fixa) são apresentados na Tabela 2. Devido a problemas no processo de amostragem móvel, os postos 4 e 6 não tiveram seus valores de BTEX determinados.

De modo geral, as concentrações encontradas através da amostragem móvel foram maiores do que na amostragem fixa. As concentrações médias de benzeno variaram de 122 a 928,3 $\mu \mathrm{g} \mathrm{m}^{-3}$ na amostragem móvel, e de $\leq \mathrm{LQ}$ a 23,3 $\mu \mathrm{g} \mathrm{m}^{-3}$ na amostragem fixa. Para o tolueno, as concentrações médias encontradas variaram de 133 a $877,2 \mu \mathrm{g} \mathrm{m}^{-3}$ na amostragem móvel, e de $\leq \mathrm{LQ}$ a $26,1 \mu \mathrm{g} \mathrm{m}^{-3}$ na amostragem fixa. Já o etilbenzeno teve concentrações que variaram de 39,1 a 128,6 $\mu \mathrm{g} \mathrm{m}^{-3}$ na amostragem móvel e de 7,6 a 17,1 $\mu \mathrm{g} \mathrm{m}^{-3}$ na amostragem fixa. Por fim, as concentrações médias totais de xilenos variaram de 136,4 a 496,8 $\mu \mathrm{g} \mathrm{m}^{-3}$ na amostragem móvel e de 5,5 a 42,9 $\mu \mathrm{g} \mathrm{m}$-3 na amostragem fixa.

As maiores concentrações médias de BTEX foram encontradas por meio da amostragem móvel, no posto 2. Quando comparadas as concentrações de BTEX entre os postos de revenda de combustíveis, nenhum dos compostos apresentou diferença estatística significativa. 
Tabela 2

Concentrações médias de benzeno, tolueno, etilbenzeno e xilenos (BTEX; $\mu \mathrm{g} \mathrm{m-3)} \mathrm{no} \mathrm{ar} \mathrm{de} \mathrm{postos} \mathrm{de} \mathrm{revenda} \mathrm{de} \mathrm{combustíveis,} \mathrm{obtidas} \mathrm{por} \mathrm{estratégias}$ de amostragem móvel e fixa, realizada no Município do Rio de Janeiro, Brasil, 2015.

\begin{tabular}{|c|c|c|c|c|c|c|c|c|c|c|}
\hline \multirow[t]{2}{*}{ Analito/Dados } & \multicolumn{4}{|c|}{ Amostragem ativa móvel } & \multicolumn{6}{|c|}{ Amostragem ativa fixa } \\
\hline & $\begin{array}{c}\text { Posto } 1 \\
(n=2)\end{array}$ & $\begin{array}{c}\text { Posto } 2 \\
(n=6)\end{array}$ & $\begin{array}{c}\text { Posto } 3 \\
(n=2)\end{array}$ & $\begin{array}{c}\text { Posto } 5 \\
(n=1)\end{array}$ & $\begin{array}{c}\text { Posto } 1 \\
(n=6)\end{array}$ & $\begin{array}{l}\text { Posto } 2 \\
(n=14)\end{array}$ & $\begin{array}{c}\text { Posto } 3 \\
(n=5)\end{array}$ & $\begin{array}{c}\text { Posto } 4 \\
(n=8)\end{array}$ & $\begin{array}{c}\text { Posto } 5 \\
(n=6)\end{array}$ & $\begin{array}{c}\text { Posto } 6 \\
(n=5)\end{array}$ \\
\hline \multicolumn{11}{|l|}{ Benzeno } \\
\hline Média & 122,0 & 928,3 & 267,3 & 187,9 & $\leq \mathrm{LQ}$ & 11,4 & $\leq \mathrm{LQ}$ & 10,2 & 23,3 & 16,7 \\
\hline Mediana & 122,0 & 290,1 & 267,3 & - & - & 2,7 & - & 6,7 & 23,9 & 15,8 \\
\hline DP & 56,1 & $1.596,0$ & 162,3 & - & - & 18,0 & - & 11,6 & 5,0 & 4,1 \\
\hline Mínimo & 82,3 & 71,2 & 152,5 & - & - & $\leq \mathrm{LQ}$ & - & $\leq \mathrm{LQ}$ & 15,2 & 10,7 \\
\hline Máximo & 161,7 & $4.168,8$ & 382,1 & - & - & 56,4 & - & 28,9 & 28,8 & 21,0 \\
\hline \multicolumn{11}{|l|}{ Tolueno } \\
\hline Média & 159,8 & 877,2 & 215,4 & 133,0 & $\leq \mathrm{LQ}$ & 17,0 & $\leq \mathrm{LQ}$ & 26,1 & 21,0 & 8,3 \\
\hline Mediana & 159,8 & 276,4 & 215,4 & - & 4,8 & 16,7 & 3,8 & 29,1 & 24,4 & 4,2 \\
\hline DP & 90,8 & $1.557,0$ & 137,7 & - & 3,0 & 12,8 & 1,0 & 21,1 & 12,0 & 6,0 \\
\hline Mínimo & 95,6 & 91,9 & 118,0 & - & $\leq \mathrm{LQ}$ & $\leq \mathrm{LQ}$ & $\leq \mathrm{LQ}$ & $\leq \mathrm{LQ}$ & $\leq \mathrm{LQ}$ & $\leq \mathrm{LQ}$ \\
\hline Máximo & 224,0 & $4.044,2$ & 312,7 & - & 9,4 & 42,8 & 5,2 & 49,3 & 34,1 & 15,5 \\
\hline \multicolumn{11}{|l|}{ Etilbenzeno } \\
\hline Média & 40,5 & 128,6 & 39,1 & 47,6 & 17,1 & 17,0 & 9,0 & 5,7 & 9,1 & 7,6 \\
\hline Mediana & 40,5 & 73,5 & 39,1 & - & 16,5 & 6,0 & 9,5 & 7,5 & 9,1 & 7,6 \\
\hline DP & 9,8 & 166,6 & 21,0 & - & 4,3 & 27,1 & 2,0 & 4,8 & 2,0 & 0,4 \\
\hline Mínimo & 33,6 & 35,3 & 24,3 & - & 11,0 & $\leq \mathrm{LQ}$ & 6,6 & $\leq \mathrm{LQ}$ & 6,7 & 7,3 \\
\hline Máximo & 47,4 & 466,4 & 54,0 & - & 23,0 & 100,4 & 11,4 & 10,6 & 11,3 & 7,9 \\
\hline \multicolumn{11}{|l|}{ Xileno (total) } \\
\hline Média & ND & 496,8 & 160,3 & 136,4 & 12,6 & 22,6 & 5,5 & 20,8 & 42,9 & 19,0 \\
\hline Mediana & ND & 161,8 & 160,3 & - & 11,6 & 15,6 & 6,0 & 18,7 & 37,1 & 19,0 \\
\hline DP & ND & 808,0 & 99,0 & - & 6,2 & 21,2 & 2,0 & 22,2 & 19,8 & 1,5 \\
\hline Mínimo & ND & 57,7 & 90,5 & - & 6,4 & $\leq \mathrm{LQ}$ & 3,3 & $\leq \mathrm{LQ}$ & 26,0 & 17,9 \\
\hline Máximo & ND & $2.136,0$ & 230,0 & - & 23,0 & 64,8 & 8,2 & 47,8 & 78,1 & 20,1 \\
\hline
\end{tabular}

DP: desvio padrão; LQ: limite de quantificação; ND: não detectado.

Nota: temperatura média de cada posto: Posto $1=22,7^{\circ} \mathrm{C}$; Posto $2=27,4^{\circ} \mathrm{C}$; Posto $3=25,4^{\circ} \mathrm{C}$; Posto $4=31,8^{\circ} \mathrm{C}$; Posto $5=31,7^{\circ} \mathrm{C}$; Posto $6=29,8^{\circ} \mathrm{C}$.

No método de amostragem fixa foi observado que a maioria das concentrações de BTEX encontradas foram um pouco acima do LQ.

Quando comparadas as concentrações de BTEX entre os postos de revenda de combustíveis, diferenças estatisticamente significativas foram encontradas para o benzeno entre o posto 5 e os postos 1 $(p=0,0030)$ e $3(p=0,0030)$; para o tolueno entre o posto 4 e os postos $1(p=0,0020), 3(p=0,0030)$ e $6(\mathrm{p}=0,0039)$; e para o xileno entre o posto 3 e os postos $4(\mathrm{p}=0,0012)$ e $5(\mathrm{p}=0,0010)$.

Ainda na Tabela 2 podem ser verificadas as temperaturas médias, nos dias de amostragem nos postos. A temperatura média entre todos os postos analisados foi de $28^{\circ} \mathrm{C}$, em coletas que ocorreram no horário da manhã até o início da tarde (entre 07:00 e 13:00), no período de fevereiro a junho. As temperaturas medidas variaram entre 21,3 (início da coleta - manhã) e $35,8^{\circ} \mathrm{C}$ (fim da coleta - tarde), entre os postos, nos dias avaliados.

A Tabela 3 apresenta os resultados dos cálculos de LADD, CR e HQ para os níveis de BTEX encontrados nas amostragens fixa e móvel.

Na Tabela 3, observa-se que os valores encontrados de CR de benzeno para trabalhadores, durante um período de exposição de 25 anos nos postos de revenda de combustíveis, mostram que para todos os postos de revenda de combustíveis analisados os valores de CR são elevados e preocupantes, principalmente no posto 2, pela amostragem móvel, que apresenta um resultado de $\mathrm{CR}=1.447 \times 10^{-6}$. 
Tabela 3

Avaliação de risco à exposição aos BTEX (benzeno, tolueno, etilbenzeno e xilenos) em trabalhadores, utilizando os dados obtidos na amostragem ativa móvel e fixa, realizada no Município do Rio de Janeiro, Brasil, 2015.

\begin{tabular}{|c|c|c|c|c|c|c|c|c|}
\hline \multirow[t]{2}{*}{ Posto/Variáveis } & \multicolumn{4}{|c|}{ Amostragem ativa móvel } & \multicolumn{4}{|c|}{ Amostragem ativa fixa } \\
\hline & Benzeno & Tolueno & Etilbenzeno & Xilenos & Benzeno & Tolueno & Etilbenzeno & Xilenos \\
\hline \multicolumn{9}{|l|}{1} \\
\hline LADD ( $\mu$ g kg-1/dia) & 6,90 & 9,12 & 2,31 & $\leq \mathrm{LQ}$ & $\leq \mathrm{LQ}$ & $\leq \mathrm{LQ}$ & 1,00 & 0,63 \\
\hline $\mathrm{CR}$ & $188,37 \times 10^{-6}$ & - & - & ND & - & - & - & - \\
\hline $\mathrm{HQ}$ & 0,80 & 0,0065 & 0,008 & ND & - & 0,0002 & 0,003 & 0,02 \\
\hline \multicolumn{9}{|l|}{2} \\
\hline LADD ( $\mu g$ kg-1/dia) & 53,00 & 50,12 & 7,34 & 28,38 & 0,65 & 1,00 & 1,00 & 0,98 \\
\hline $\mathrm{CR}$ & $1.447 \times 10^{-6}$ & - & - & - & $2,08 \times 10^{-6}$ & - & - & - \\
\hline $\mathrm{HQ}$ & 6,19 & 0,035 & 0,025 & 0,97 & 0,07 & 0,0007 & 0,003 & 0,03 \\
\hline \multicolumn{9}{|l|}{3} \\
\hline LADD ( $\mu$ g kg-1/dia) & 15,27 & 12,30 & 2,23 & 9,16 & $\leq \mathrm{LQ}$ & $\leq \mathrm{LQ}$ & 0,51 & 0,3 \\
\hline $\mathrm{CR}$ & $416,8 \times 10^{-6}$ & - & - & - & - & - & - & - \\
\hline HQ & 1,78 & 0,0087 & 0,008 & 0,31 & - & - & 0,001 & 0,01 \\
\hline \multicolumn{9}{|l|}{4} \\
\hline LADD ( $\mu g$ kg-1/dia) & - & - & - & - & 0,58 & 1,50 & 0,32 & 2,36 \\
\hline$C R$ & - & - & - & - & $15,8 \times 10-6$ & - & - & - \\
\hline $\mathrm{HQ}$ & - & - & - & - & 0,07 & 0,001 & 0,001 & 0,08 \\
\hline \multicolumn{9}{|l|}{5} \\
\hline LADD ( $\mu g$ kg-1/dia) & 10,73 & 7,60 & 2,72 & 7,80 & 1,33 & 1,20 & 0,50 & 2,18 \\
\hline CR & $293 \times 10^{-6}$ & - & - & - & $36,3 \times 10-6$ & - & - & - \\
\hline $\mathrm{HQ}$ & 1,25 & 0,005 & 0,01 & 0,26 & 0,15 & 0,0008 & 0,001 & 0,07 \\
\hline \multicolumn{9}{|l|}{6} \\
\hline LADD ( $\left.\mu g \mathrm{~kg}^{-1} / \mathrm{dia}\right)$ & - & - & - & - & 1,00 & 0,47 & 0,43 & 0,77 \\
\hline $\mathrm{CR}$ & - & - & - & - & $27,3 \times 10^{-6}$ & - & - & - \\
\hline HQ & - & - & - & - & 0,11 & 0,0003 & 0,0015 & 0,03 \\
\hline
\end{tabular}

CR: risco de câncer; HQ: quociente de perigo; LADD: dose média diária da exposição; LQ: limite de quantificação; ND: não detectado.

Continuando com a Tabela 3, todos os compostos, com exceção do benzeno, apresentaram valores do $\mathrm{HQ}$ abaixo de $1(\mathrm{HQ}<1)$. Isso significa que não se apresenta nenhum efeito adverso aparente à saúde dos trabalhadores durante a exposição. Já para a exposição ao benzeno, podemos observar que os valores de HQ foram acima de 1 (HQ > 1) nos postos 2, 3 e 5 , mostrando que nesses postos podem ocorrer efeitos adversos à saúde dos trabalhadores.

\section{Discussão}

Os valores apresentados na Tabela 2 da amostragem ativa móvel devem ser analisados considerando que os trabalhadores participantes deste estudo exercem diversas funções dentro de um postos de revenda de combustíveis, e assim, sua exposição aos vapores da gasolina durante o trabalho é variável, uma vez que eles se movimentam pelo espaço do posto. Desta forma, este tipo de estratégia amostral mostrou-se mais adequada para avaliar os níveis de BTEX a que o trabalhador está exposto, tendo em vista que os postos apresentam locais com diferentes concentrações de BTEX. Isso se confirma quando observamos os maiores resultados na amostragem móvel. Já os resultados da amostragem fixa mostram a exposição ao BTEX a partir da proximidade das bombas de combustível.

A grande variação nas concentrações de BTEX obtidas nos postos pode ocorrer, em ambos os casos, devido a alguns fatores, tais como: os postos ficam perto de rodovias e o fluxo de automóveis 
contribui para elevação de BTEX no ar, variando ao longo do dia; o fluxo de vento nos locais amostrados também varia bastante no decorrer do tempo; a quantidade de abastecimentos realizados durante a jornada de trabalho influencia nas concentrações de BTEX, pois nesse momento o trabalhador tem mais contato com os compostos da gasolina; e, também é importante considerar que as coletas ocorreram entre 7 horas da manhã até 13 horas da tarde, e nesse intervalo de tempo há um aumento da temperatura, que contribui para uma maior volatilização dos compostos, influenciando os resultados.

De acordo com Carrieri et al. 21, a população como um todo está exposta ambientalmente ao BTEX, já que ocorre a volatilização dos solventes presentes na gasolina oriunda dos postos de revenda de combustíveis e das emissões dos veículos, levando à dispersão generalizada desses vapores para a atmosfera.

Os valores de benzeno da amostragem ativa fixa apresentaram-se estatisticamente diferentes entre alguns postos. Uma possível justificativa para esses resultados seria a diferença de circulação de ar nesses postos de revenda de combustíveis, devido às suas localizações, pois alguns estavam localizados em campo aberto, com grande circulação de ar e vento, enquanto outros estavam localizados em centros urbanos com construções ao redor, que servem como obstáculo para a circulação do ar. Outra justificativa seria que os postos de revenda de combustíveis de grande porte e/ou com grande volume de vendas possuem maior emissão de vapores de benzeno.

Comparando as concentrações médias de BTEX encontradas neste estudo - para a amostragem ativa móvel - com os limites estabelecidos pela Norma Regulamentadora no 15 (NR-15) 22 e pela metodologia no 1.501 do NIOSH 17, podem ser feitas algumas observações, sendo a principal que apenas o benzeno ultrapassou os limites preconizados.

Para o benzeno as concentrações mínimas e máximas foram de 122 e 928,3 $\mu \mathrm{g} \mathrm{m}^{-3}$, respectivamente. A NR-15 22 recomenda um valor de referência tecnológico (VRT), que é a "concentração de benzeno no ar considerada exequível do ponto de vista técnico, definido em processo de negociação tripartite" para empresas petroquímicas, não aplicável a postos de revenda de combustíveis, de $3.190 \mu \mathrm{g} \mathrm{m}^{-3}$, porém, destaca que "o cumprimento do VRT é obrigatório e não exclui risco à saúde". Em recente atualização de 2016, a NR-9 (Anexo II) 18 traz uma série de requisitos mínimos de segurança e saúde no trabalho em postos de revenda de combustíveis, porém, não estipula um limite de exposição.

Devido a essa complexidade e riscos relacionados ao benzeno, por ser um carcinogênico, qualquer concentração pode levar a danos à saúde 23. Esses aspectos evidenciam que o limite para exposições ao benzeno deveria ser zero, ou o mais próximo possível disso. Já o limite estipulado pelo NIOSH é de $320 \mu \mathrm{g} \mathrm{m}^{-3}$, mostrando que para o benzeno as concentrações estão acima dos limites, o que leva a uma contribuição no aumento do risco de câncer nos trabalhadores. Este valor do NIOSH também mostra como nossa legislação ainda aceita valores altíssimos para exposição ao benzeno, uma vez que este limite é dez vezes menor que o VRT.

Para o tolueno as concentrações mínimas e máximas foram de 133 e 877,2 $\mu \mathrm{g} \mathrm{m}^{-3}$, respectivamente, sendo que o limite estipulado pela NR-15 é de $290.000 \mu \mathrm{g} \mathrm{m}^{-3}$ e pelo NIOSH é de $375.000 \mu \mathrm{g} \mathrm{m}^{-3}$, podendo-se observar que o composto tolueno não excedeu os limites recomendados.

O etilbenzeno apresentou concentrações mínimas e máximas de 39,1 e 128,6 $\mu \mathrm{g} \mathrm{m}^{-3}$, respectivamente. O limite pela NR-15 é de $340.000 \mu \mathrm{g} \mathrm{m}-3$, e pelo NIOSH é de $435.000 \mu \mathrm{g} \mathrm{m}^{-3}$. Observa-se que o etilbenzeno não apresentou concentrações acima dos limites preconizados.

O xileno apresentou concentrações mínimas e máximas de 136,4 e 496,8 $\mu \mathrm{g} \mathrm{m} \mathrm{m}^{-3}$, respectivamente. O limite pela NR-15 é de $340.000 \mu \mathrm{g} \mathrm{m}^{-3}$, e pelo NIOSH é de $435.000 \mu \mathrm{g} \mathrm{m}^{-3}$. Desta forma, o xileno não apresentou concentrações acima dos limites estabelecidos.

Os valores de HQ devem ser analisados de forma que se o resultado da razão for maior do que 1, o ambiente de trabalho pode levar a prováveis efeitos adversos à saúde do trabalhador, o que é evidenciado nos postos em que o HQ foi calculado para o benzeno, nos quais os resultados foram altos. Sendo um composto comprovadamente carcinogênico, esses valores elevados representam um perigo à saúde dos trabalhadores.

Com o objetivo de comparar os resultados encontrados neste estudo e em outros realizados em diferentes cidades do Brasil, assim como em outros países, a Tabela 4 apresenta trabalhos nacionais e internacionais que avaliaram BTEX em postos de revenda de combustíveis, por amostragem fixa.

O estudo realizado por Santos 1 em Amargosa (Bahia), em cinco postos de revenda de combustíveis com amostragem ativa e estratégia metodológica parecida com o presente estudo, encontrou 
Tabela 4

Concentrações médias de benzeno, tolueno, etilbenzeno e xilenos (BTEX; $\mu \mathrm{g}$ m-3), obtidas por amostragem ativa fixa, comparadas com estudos nacionais e internacionais.

\begin{tabular}{|c|c|c|c|c|c|}
\hline Estudo (ano) & Localidade & Benzeno & Tolueno & Etilbenzeno & Xilenos \\
\hline \multicolumn{6}{|l|}{ Nacionais } \\
\hline Santos 1 (2014) & Postos; Amargosa (Bahia) & 24,9 & 106,6 & 18,6 & 78,2 \\
\hline Correa et al. 24 (2012) & Postos em área urbana; Rio de Janeiro & 29,7 & 47,7 & 23,3 & 61,2 \\
\hline Grosjean et al. 36 (1999) & $\begin{array}{l}\text { Rodovia em área urbana; Porto Alegre } \\
\text { (Rio Grande do Sul) }\end{array}$ & 24,9 & 43,1 & 13,7 & 39,0 \\
\hline Oliveira et al. 37 (2007) & Postos; Niterói (Rio de Janeiro) & 262,0 & 299,0 & 80,0 & 259,0 \\
\hline Cruz et al. 2 (2017) & Postos; Salvador (Bahia) & 211,9 & 107,5 & 17,5 & 26,5 \\
\hline Este trabalho & Postos; Rio de Janeiro & 15,4 & 18,1 & 10,9 & 20,5 \\
\hline \multicolumn{6}{|l|}{ Internacionais } \\
\hline Esmaelnejad et al.10 (2015) & Postos; Shahreza (Irã) & $268,5 / 213,5$ * & $442,2 / 402,5$ * & $100,6 / 90,8$ * & $360,2 / 439,2$ * \\
\hline Lan et al. 26 (2013) & $\begin{array}{l}\text { Rodoviária em área urbana; Ho Chi Minh } \\
\text { (Vietnã) }\end{array}$ & 56,0 & 121,0 & 21,0 & 87,0 \\
\hline Majumdar et al. 27 (2011) & Área urbana e comercial; Calcutá (Índia) & 29,2 & 45,4 & 13,1 & 44,8 \\
\hline Brocco et al. 28 (1996) & Rodovia em área urbana: Roma (Itália) & 35,5 & 99,7 & 17,6 & 79,7 \\
\hline Khoder et al. 29 (2007) & Postos; Cairo (Egito) & 87,2 & 213,8 & 43,3 & 214,6 \\
\hline Kim et al. 25 (2001) & Postos; Birmingham (Inglaterra) & 7,3 & 15,1 & 1,6 & 6,6 \\
\hline
\end{tabular}

*Amostras coletadas no inverno e verão, respectivamente.

concentrações médias de benzeno próximas às nossas, enquanto as concentrações do tolueno foram aproximadamente seis vezes maiores do que as nossas.

Comparando com o estudo realizado por Correa et al. 24 - em 11 postos de revenda de combustíveis no Rio de Janeiro, utilizando o método de amostragem ativa próxima as bombas, e amostras coletadas durante um período de 20 minutos - foram encontradas as concentrações um pouco maiores. Mesmo com nossos valores estando próximos a este estudo, a estratégia de amostragem realizada no trabalho de Correa et al. teve uma duração de 20 minutos, enquanto neste trabalho a coleta durou 5 horas, desta forma, pode-se presumir que as concentrações de BTEX no ar dos postos estudados por Correa et al. deveriam ser maiores.

No estudo realizado por Esmaelnejad et al. 10, a amostragem do ar para determinação de BTEX foi realizada em bombas de 8 postos de revenda de combustíveis em Shahreza (Irã), com coletas no inverno e no verão. Como pode ser observado a variação dos climas interfere nos valores, pois os resultados encontrados por Esmaelnejad et al. foram muito maiores do que nesse estudo, para todos os BTEX, em ambas as estações.

Os valores encontrados neste trabalho são maiores do que em Birmingham (Inglaterra) 25, que é também, comparativamente, o estudo com as menores concentrações de BTEX em postos de revenda de combustíveis reportado na literatura aqui discutida. Porém, essas concentrações baixas no estudo inglês podem ser explicadas pela baixa temperatura do clima da cidade de Birmingham, o que diminui a evaporação dos compostos, levando a concentrações de BTEX mais baixas. Esta não é a mesma realidade que encontramos no clima brasileiro, e, portanto, os outros estudos (Vietnã 26, Índia 27 e Itália ${ }^{28)}$ provavelmente devem ter condições climáticas mais semelhantes às nossas. Além disso, estes outros estudos avaliaram BTEX em áreas urbanas, comerciais e rodovias, o que também influencia nos níveis encontrados.

O estudo de Khoder et al. 29 em postos de revenda de combustíveis também apresentou altas concentrações de BTEX em comparação aos outros estudos analisados. Realizado no Cairo (Egito), que assim como o estudo do Irã, é um local que apresenta temperaturas médias mais elevadas, levando ao aumento da evaporação dos compostos do BTEX, aumentando a concentração destes na atmosfera 10. 
Essas variações também podem ser atribuídas às diferenças na composição das gasolinas utilizadas em outros países, além dos fatores sazonais e meteorológicos de cada local.

As diferenças entre as concentrações encontradas nos trabalhos internacionais e este estudo podem ser explicadas não apenas por serem de localidades e ambientes distintos, mas também por serem locais com temperaturas ambientes distintas, o que pode influenciar os níveis de BTEX no ar. Este estudo apresentou uma temperatura média de $28^{\circ} \mathrm{C}$, nos dias de amostragem nos postos.

A comparação entre estudos é difícil de se realizar, já que consistem em diferentes condições experimentais, métodos de amostragem e análises que podem justificar as diferenças, muitas vezes, discrepantes, entre os valores.

As diferenças que podem ser observadas nas concentrações médias dos estudos internacionais, em relação a este estudo que se dá na cidade do Rio de Janeiro, podem estar relacionadas, como sugere o estudo de Cruz et al. 2, a composição da gasolina brasileira, que é misturada com 27\% v/v de etanol anidro, o que contribui para mudar a volatilidade do BTEX, e reduzir a emissão destes compostos para atmosfera.

As concentrações médias de BTEX encontradas neste estudo foram relevantes e seus níveis são uma ameaça para a saúde dos trabalhadores. Uma das ameaças à saúde é o risco de desenvolvimento de câncer, sendo um dos efeitos toxicológicos mais perigosos do BTEX, principalmente o benzeno. É clássico na literatura que indivíduos expostos ocupacionalmente ao benzeno apresentam um risco aumentado no desenvolvimento de leucemia 3,30,31.

Através do uso de modelos matemáticos, que foram baseados em estudos em humanos e animais, é possível estimar a probabilidade de uma pessoa vir a desenvolver um câncer por respirar ar contendo concentrações específicas de compostos químicos 13,19.

Analisando os resultados da Tabela 3 podemos observar que os resultados de CR (benzeno) obtidos neste estudo foram muito altos em todos os postos, pois estão bem acima do limite estabelecido pela USEPA 32, de 1,00×10-6, o que torna necessário a adoção de medidas que protejam a saúde dos trabalhadores, tais como mudanças no processo e ambiente de trabalho, uso de equipamentos de proteção individual, supervisão de agências responsáveis e o monitoramento de exposição.

Para os limites de exposição ocupacional, o grupo European Scientific Expert estima que o valor limite de exposição ocupacional ao benzeno de $1.600 \mu \mathrm{g} \mathrm{m}^{-3}$ reduziria o risco de vida útil em aproximadamente até 0,25-3,3 em casos adicionais de leucemia por 1.000 trabalhadores expostos 15. A relativa exposição a agentes cancerígenos no local de trabalho estabelece um valor limite de exposição ao benzeno de $3.250 \mu \mathrm{g} \mathrm{m}^{-3}$, durante oito horas de trabalho por dia. Devido à pressão feita pelos setores industriais houve uma mudança, para atividades selecionadas, nas quais poderia ser permitido um valor limite máximo de $9.750 \mu \mathrm{g} \mathrm{m} \mathrm{m}^{-3}$. Mas mesmo mantendo um valor de $3.250 \mu \mathrm{g} \mathrm{m}^{-3}$ ainda se tem um risco considerável de mortalidade na vida de um trabalhador 15 , sendo um equívoco seguir essa linha de pensamento, pois, por mais que se diminua os limites de exposição para benzeno, não existe limite seguro para o mesmo 33.

A Figura 1 apresenta dois gráficos que mostram o CR em cada um dos postos de revenda de combustíveis avaliados neste estudo, para a amostragem móvel e fixa, comparados com o CRVL de $1,00 \times 10-6$.

Analisando os dados obtidos nas amostragens móvel e fixa, em comparação com o CRLV, pode-se observar que todos os postos que apresentaram resultados do cálculo de risco de câncer estavam acima do limite estipulado, mostrando que os riscos de desenvolver câncer nos trabalhadores são muito altos, o que torna necessário medidas que minimizem a exposição, reduzindo os riscos.

A definição de valores limite nas legislações para exposição ao benzeno deveria ser revista, já que não existe concentração segura para exposição a esta substância, pois durante uma exposição em longo prazo, mesmo em concentrações baixas, há riscos à saúde, principalmente de efeitos sistêmicos e carcinogênicos 20,23,33,34,35. 
Figura 1

Risco de câncer (CR) de cada posto estudado, obtidos através da amostragem ativa móvel e fixa, realizada no Município do Rio de Janeiro, Brasil, 2015.

1a) Amostragem ativa móvel

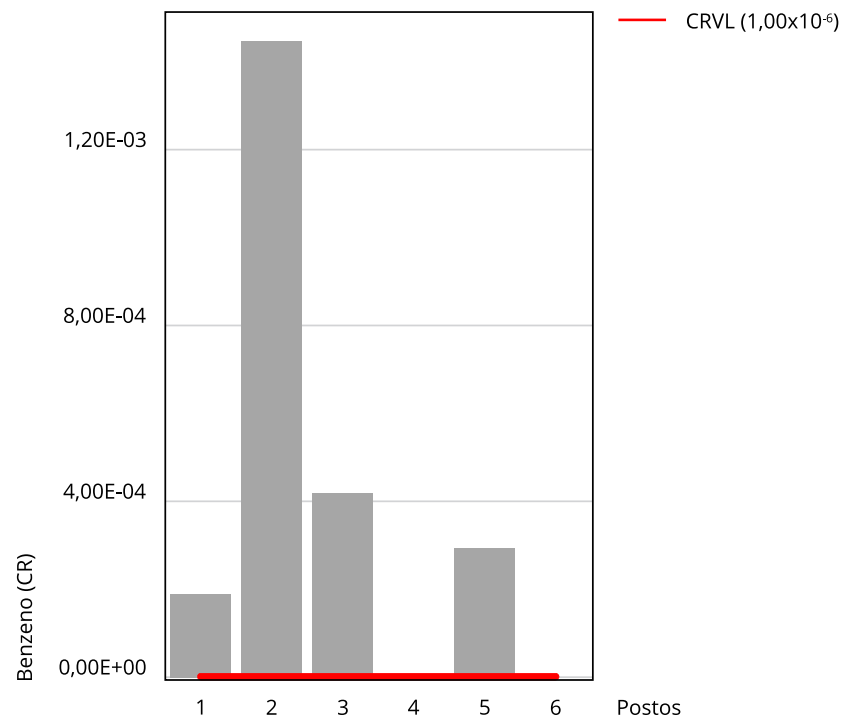

1b) Amostragem ativa fixa

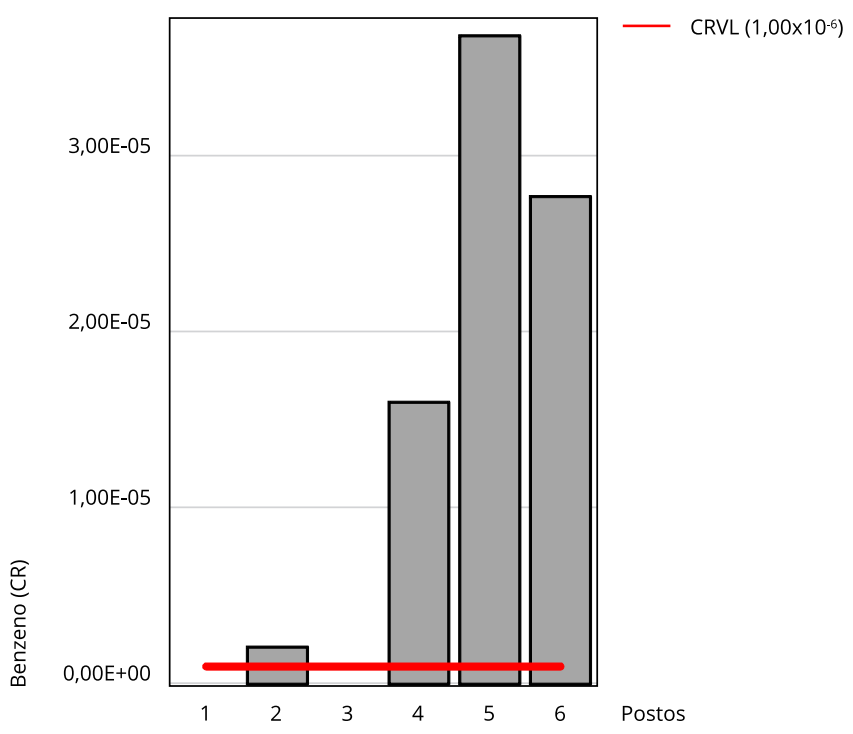

CRVL: valor limite para risco de câncer.

\section{Conclusão}

Os resultados encontrados nas amostragens móvel e fixa foram, em sua maioria, valores acima dos limites de quantificação. As concentrações de BTEX se mantiveram dentro dos limites preconizados, exceto para o benzeno, mostrando que os postos de revenda de combustíveis são locais de trabalho potencialmente perigosos. Mesmo que alguns compostos estejam dentro dos valores recomendados, a exposição a esses compostos, de forma contínua, principalmente ao benzeno, pode vir a promover danos a longo prazo, como o câncer.

Os resultados de HQ para o benzeno foram elevados em três postos, mostrando que pode haver efeitos adversos à saúde dos trabalhadores nesses locais. Em relação ao CR para o benzeno, os valores, em todos os postos e em ambas as amostragens, foram acima dos indicados pela USEPA.

Diante desses dados, mostra-se que os trabalhadores de postos de revenda de combustíveis são uma população com riscos à saúde, indicando a necessidade de mudanças nesse processo e ambiente de trabalho, devido à exposição a compostos carcinogênicos, para os quais não deveriam existir limites considerados seguros nas legislações. 


\section{Colaboradores}

V. O. Figueiredo contribuiu com a interpretação dos resultados e redação do artigo e é responsável por todos os aspectos do trabalho na garantia da exatidão e integridade de qualquer parte da obra. S. R. Alves contribuiu com a interpretação dos resultados, redação do artigo e revisão crítica relevante do conteúdo intelectual. L. V. B. Carvalho, R. M. Borges, I. C. Costa-Amaral e A. L. Larentis contribuíram com o planejamento da pesquisa, coleta das amostras em campo, interpretação dos dados, redação do artigo e revisão crítica relevante do conteúdo intelectual. M. V. C. Santos, A. C. S. Rosa, R. C. O. C. Mattos e P. N. Sarcinelli contribuíram com a interpretação e revisão dos dados utilizados no artigo e concepção e projeto do estudo. M. A. C. Menezes contribuiu com o planejamento da pesquisa, análise dos dados e redação do artigo. E. S. Gonçalves contribuiu com o planejamento da pesquisa, interpretação dos resultados e redação do artigo. Todos os autores aprovaram a versão final.

\section{Informações adicionais}

ORCID: Victor Oliva Figueiredo (0000-0002-26565494); Leandro Vargas Barreto de Carvalho (00000002-3090-7477); Renato Marçullo Borges (00000003-3170-3772); Isabele Campos Costa-Amaral (0000-0003-3358-7381); Marcus Vinicius Corrêa dos Santos (0000-0001-7096-5156); Ana Cristina Simões Rosa (0000-0003-0547-1138); Marco Antônio Carneiro de Menezes (0000-0002-79476639); Rita de Cássia Oliveira da Costa Mattos (0000-0002-0523-7467); Paula Novaes Sarcinelli (0000-0002-8761-9142); Sergio Rabello Alves (0000-0002-4181-1163); Ariane Leites Larentis (0000-0001-7232-3245); Eline Simões Gonçalves (0000-0001-6842-3016).

\section{Agradecimentos}

Agradecemos à Escola Nacional de Saúde Pública Sergio Arouca, Fundação Oswaldo Cruz (Inova-Ensp), à Coordenação de Aperfeiçoamento de Pessoal de Nível Superior (CAPES) e à Fundação de Amparo à Pesquisa do Estado do Rio de Janeiro (FAPERJ).

\section{Referências}

1. Santos GLG. Desenvolvimento e aplicação de método de dessorção química para determinação de BTEX em ar ambiente de postos de combustíveis utilizando cromatografia a gás [Tese de Doutorado]. Salvador: Universidade Federal da Bahia; 2014.

2. Cruz LPS, Alves LP, Santos AVS, Esteves MB, Gomes ÍVS, Nunes LSS. Assessment of BTEX concentrations in air ambient of gas stations using passive sampling and the health risks for workers. J Environ Prot 2017; 8:12-25.

3. Amaral ICC, Carvalho LVB, Pimentel JNS, Pereira AC, Vieira JA, Castro VS, et al. Avaliação ambiental de BTEX (benzeno, tolueno, etilbenzeno, xilenos) e biomarcadores de genotoxicidade em trabalhadores de postos de combustíveis. Rev Bras Saúde Ocup 2017; 42 Suppl $1: e 8 s$.

4. Tunsaringkarn T, Siriwong W, Rungsiyothin A, Nopparatbundit S. Occupational exposure of gasoline station workers to BTEX compounds in Bangkok, Thailand. Int J Occup Environ Med 2012; 3:117-25.

5. Campos MAA, Fernandes APSM, André LC. Avaliação da exposição ocupacional ao benzeno em trabalhadores frentistas e analistas de combustíveis utilizando o Teste Cometa como biomarcador de genotoxicidade. Rev Bras Saúde Ocup 2017; 42 Suppl 1:e6s.

6. Moriyama INH, Pinto VRS, Santana LG, Pinto AC, Poldi RMV, Almeida IM. Prevenção da exposição ocupacional ao benzeno em trabalhadores de postos de revenda de combustíveis: a experiência do estado do Espírito Santo. Rev Bras Saúde Ocup 2017; 42 Suppl 1:e4s.

7. Buczynska AJ, Krata A, Stranger M, Locateli Godoi AF, Kontozova-Deutsch V, Bencs L, et al. Atmospheric BTEX-concentrations in an area with intensive street traffic. Atmos Environ 2009; 43:311-8.

8. Giardini I, Poça KS, Silva VSP, Mello MSC, Friedrich K. Vigilância sanitária em postos de revenda de combustíveis: aplicação de um modelo para integrar ações e promover a saúde do trabalhador. Rev Bras Saúde Ocup 2017; 42 Suppl 1:e7s.

9. Arêas JS, Pinheiro CESC, Santelli RE, Machado W, Bielschowsky C, Rocha RT, et al. Seriam as áreas contaminadas do Estado do Rio de Janeiro um legado da grande aceleração no Antropoceno? Revista Virtual de Química 2020; 12:775-94

10. Esmaelnejad F, Hajizadeh Y, Pourzamani H, Amin M. Monitoring of benzene, toluene, ethyl benzene, and xylene isomers emission from Shahreza gas stations in 2013. Int J Environ Health Eng 2015; 4:17.

11. Barata-Silva C, Mitri S, Pavesi T, Saggioro E, Moreira JC. Benzeno: reflexos sobre a saúde pública, presença ambiental e indicadores biológicos utilizados para a determinação da exposição. Cad Saúde Colet (Rio J.) 2014; 22:329-42. 
12. American Conference of Governmental Industrial Hygienists. 2012 TLVs and BEIs: based on the documentation of the threshold limit values for chemical substances and physical agents and biological exposure indices. Cincinnati: American Conference of Governmental Industrial Hygienists; 2012.

13. International Agency for Research on Cancer. Benzene. Lyon: International Agency for Research on Cancer; 2019. (IARC Monographs on the Evaluation of the Carcinogenic Risk of Chemicals to Humans, 120).

14. World Health Organization. Guidelines for air quality. Geneva: World Health Organization; 2000.

15. Periago JF, Prado C. Evolution of occupational exposure to environmental levels of aromatic hydrocarbons in service stations. Ann Occup Hyg 2005; 49:233-40.

16. Secretaria Municipal do Meio Ambiente. Dados da cidade. http://www.rio.rj.gov.br/web/ smac/ (acessado em 12/Out/2017).

17. National Institute for Occupational Safety and Health. Hidrocarbons, Aromatic: Method 1501. In: NIOSH Manual of Analytical Methods. 4th Ed. Washington DC: National Institute for Occupational Safety and Health; 2003. https://www.cdc.gov/niosh/docs/2003-154/ default.html.

18. Ministério do Trabalho e Emprego. Portaria no 1.109 , de 21 de setembro de 2016. Aprova o Anexo 2 - Exposição Ocupacional ao Benzeno em Postos Revendedores de Combustíveis - PRC - da Norma Regulamentadora no 9 Programa de Prevenção de Riscos Ambientais - PPRA. Diário Oficial da União 2016; 22 set.

19. Edokpolo B, Yu Q, Connell D. Health risk assessment of ambient air concentrations of benzene, toluene and xylene (BTX) in service station environments. Int J Environ Res Public Health 2014; 11:6354-74.

20. Valente D, Costa-Amaral IC, Carvalho LVB, Santos MVC, Castro VS, Rodrigues DRF, et al. Utilização de biomarcadores de genotoxicidade e expressão gênica na avaliação de trabalhadores de postos de combustíveis expostos a vapores de gasolina. Rev Bras Saúde Ocup 2017; 42 Suppl 1:e2s.

21. Carrieri M, Bonfiglio E, Scapellato ML, Maccà I, Tranfo G, Faranda P, et al. Comparison of exposure assessment methods in occupational exposure to benzene in gasoline filling-station attendants. Toxicol Lett 2006; 162:146-52.

22. Ministério do Trabalho e Emprego. Portaria no 14, de 20 de dezembro de 1995. Altera a redação do item "Substâncias Cancerígenas" do Anexo XIII da Norma Regulamentadora NR15 - Atividades e Operações Insalubres - e inclui o Anexo XIII-A "Benzeno”. Diário Oficial da União 1995; 22 dez.

23. Mendes M, Machado JMH, Durand A, Costa-Amaral IC, Valente D, Gonçalves ES, et al. Normas ocupacionais do benzeno: uma abordagem sobre o risco e exposição nos postos de revenda de combustíveis. Rev Bras Saúde Ocup 2017; 42 Suppl 1:e3s.
24. Correa SM, Arbilla G, Marques MRC, Oliveira KMPG. The impact of BTEX emissions from gas stations into the atmosphere. Atmos Pollut Res 2012; 3:163-9.

25. Kim YM, Harrad S, Harrison RM. Concentrations and sources of VOCs in urban domestic and public microenvironments. Environ Sci Technol 2001; 35:997-1004.

26. Lan TTN, Minh PA. BTEX pollution caused by motorcycles in the megacity of Ho Chi Minh. J Environ Sci 2013; 25:348-56.

27. Majumdar D, Mukherjeea AK, Sen S. BTEX in ambient air of a metropolitan city. J Environ Prot 2011; 2:11-20.

28. Brocco D, Fratarcangeli R, Lepore L, Petricca $\mathrm{M}$, Ventrone I. Determination of aromatic hydrocarbons in urban air of Rome. Atmos Environ 1997; 31:557-66.

29. Khoder MI. Ambient levels of volatile organic compounds in the atmosphere of Greater Cairo. Atmos Environ 2007; 41:554-66.

30. Costa DF, Goldbaum M. Contaminação química, precarização, adoecimento e morte no trabalho: benzeno no Brasil. Ciênc Saúde Colet 2017; 22:2681-92.

31. Bausà R, Navarro L, Cortès-Franch I. Myelofibrosis in a benzene-exposed cleaning worker. Arch Prev Riesgos Labor 2017; 20:167-9.

32. U.S. Environmental Protection Agency. Methods for derivation of inhalation reference concentrations and application of inhalation dosimetry. Washington DC: U.S. Environmental Protection Agency; 1994.

33. Larentis AL, Carvalho LVB, Gonçalves ES, Costa-Amaral IC. Crítica à abordagem toxicológica nas avaliações de exposições de trabalhadores a substâncias químicas a partir da perspectiva do modelo operário italiano (MOI). In: Oddone I, Marri G, Gloria S, Briante $\mathrm{G}$, Chiatella M, Re A, organizadores. Ambiente de trabalho: a luta dos trabalhadores pela saúde. 2a Ed. São Paulo: Hucitec Editora; 2020. p. 217-33.

34. Costa-Amaral IC, Carvalho LVB, Santos MVC, Valente D, Pereira AC, Figueiredo VO, et al. Environmental assessment and evaluation of oxidative stress and genotoxicity biomarkers related to chronic occupational exposure to benzene. Int J Environ Res Public Health 2019; 16:2240.

35. Poça KS, Giardini I, Silva PVB, Geraldino BR, Bellomo A, Alves JA, et al. Gasoline-station workers in Brazil: benzene exposure; genotoxic and immunotoxic effects. Mutat Res Genet Toxicol Environ Mutagen 2021; 865:503322.

36. Grosjean E, Rasmussen RA, Grosjean D. Toxic air contaminants in Porto Alegre, Brazil. Environ Sci Technol 1999; 33:1970-8.

37. Oliveira KMPG, Arbilla G, Silva LSV. Monitoramento de BTEX em um posto de combustíveis na cidade de Niterói. In: Resumos do XI Encontro da Sociedade Brasileira de Química-Regional Rio de Janeiro. Rio de Janeiro: Sociedade Brasileira de Química-Regional Rio de Janeiro; 2007. p. 29-30. 
Abstract

Gasoline is a complex mixture of substances, including aromatic hydrocarbons such as benzene, toluene, ethylbenzene, and xylenes (BTEX). These compounds are emitted into the air, with the special relevance of benzene since it is provenly carcinogenic. The study aimed to assess BTEX concentrations in filling stations in the city of Rio de Janeiro, Brazil, and to calculate the cancer risk associated with such exposures. Two types of sampling were performed (stationary and mobile), adapted from methodology n. 1,501 (U.S. National Institute for Occupational Safety and Health) for aromatic hydrocarbons, in six filling stations in the West Zone of Rio de Janeiro. Stationary sampling was done near the fuel pumps, while mobile sampling was done in the breathing zone of the workers (station attendants) as they moved around the station. The samples were analyzed with gas chromatography flame ionization detector. The sampling results were used to calculate the health risk, using the indicators Hazard quotient (HQ) and Cancer risk (CR) to assess the possible noncarcinogenic and carcinogenic effects, respectively, in filling station workers. Environmental concentrations for the most of the BTEX compounds were below the recommended limits, except for benzene, a carcinogenic compound, which displayed concentrations far above the limits, leading to high cancer risk values. The results showed that there are health risks for filling station attendants, especially the risk of developing cancer from excessive exposure to benzene.

Occupational Health; Benzene; Filling Station; Carcinogens

\section{Resumen}

La gasolina es una mezcla compleja de sustancias, entre ellas existen hidrocarburos como el benceno, tolueno, etilbenceno y xilenos (BTEX), emitiéndose estos compuestos a la atmósfera, donde se destaca el benceno, al tratarse de un carcinogénico comprobado. Los objetivos fueron evaluar las concentraciones de BTEX en el aire de puestos de reventa de combustibles, en el municipio de Río de Janeiro, Brasil, así como calcular el riesgo de cáncer asociado a estas exposiciones. Se realizaron dos tipos de muestra (fija y movible), adaptadas de la metodología no 1.501 del Instituto Nacional de Seguridad y Salud Laboral (EE.UU.), en seis puestos de reventa de combustibles de la zona oeste de la ciudad. La muestra fija se realizó cerca de las bombas de combustible, mientras que la móvil se hizo mediante tomas de aire en la zona respiratoria de los trabajadores (de la gasolinera), a medida que estos se desplazaban por el lugar de trabajo. Las muestras se analizaron por cromatografía gaseosa con detección por ionización en llama. Junto a los resultados de las muestras, se realizaron cálculos de riesgo para la salud, usando los indicadores Cociente de peligro (HQ) y Riesgo de cáncer (CR), con el fin de evaluar los posibles efectos no-carcinogénicos y carcinogénicos, respectivamente, en los trabajadores de los puestos de reventa de combustibles. Las concentraciones ambientales para la mayoría de los compuestos BTEX estuvieron por debajo de los limites preconizados, menos en el caso del benceno, un compuesto carcinogénico, que presentó concentraciones muy por encima de los límites, llevando a altos valores de riesgo de cáncer. Los resultados demostraron que existen riesgos para la salud de los trabajadores de puestos de reventa de combustibles, principalmente, riesgo de desarrollar cáncer, debido a la exposición excesiva al benceno.

Salud Laboral; Benceno; Gasolineras; Carcinógenos
Recebido em 30/Dez/2020

Versão final reapresentada em 13/Mar/2021

Aprovado em 15/Abr/2021 